\title{
Angular momentum loss and stellar spin-down in magnetic massive stars
}

\author{
Asif ud-Doula ${ }^{1}$, Stanley P. Owocki ${ }^{2}$ and Richard H.D. Townsend ${ }^{3}$ \\ ${ }^{1}$ Department of Physics, SUNY-Morrisville State College, Morrisville, NY 13408, USA \\ e-mail: uddoula@morrisville.edu \\ ${ }^{2}$ Department of Physics and Astronomy, University of Delaware, \\ Newark, DE 19716, USA \\ ${ }^{3}$ Department of Physics, University of Wisconsin-Madison, \\ Madison, WI 53706, USA
}

\begin{abstract}
We examine the angular momentum loss and associated rotational spin-down for magnetic hot stars with a line-driven stellar wind and a rotation-aligned dipole magnetic field. Our analysis here is based on our previous 2-D numerical MHD simulation study that examines the interplay among wind, field, and rotation as a function of two dimensionless parameters, $\mathrm{W}\left(=\right.$ Vrot/Vorb) and 'wind magnetic confinement', $\eta_{*}$ defined below. We compare and contrast the 2-D, time variable angular momentum loss of this dipole model of a hot-star wind with the classical 1-D steady-state analysis by Weber and Davis (WD), who used an idealized monopole field to model the angular momentum loss in the solar wind. Despite the differences, we find that the total angular momentum loss averaged over both solid angle and time follows closely the general WD scaling $\dot{J} \sim \dot{M} \Omega R_{A}^{2}$. The key distinction is that for a dipole field Alfvèn radius $R_{A}$ is significantly smaller than for the monopole field WD used in their analyses. This leads to a slower stellar spin-down for the dipole field with typical spin-down times of order 1 Myr for several known magnetic massive stars.
\end{abstract}

Keywords. MHD - Stars: winds - Stars: magnetic fields - Stars: early-type - Stars: rotation Stars: mass loss

\section{Introduction}

An outflowing wind carries away angular momentum and thus spins down the stellar rotation. Winds with magnetic fields exert a braking torque that is significantly larger than for non-magnetic cases, due to the larger lever arm of magnetic field lines that extend outward from the stellar surface. A seminal analysis of this process was carried out by Weber \& Davis (WD, 1967), who modelled the angular momentum loss of the solar wind for the idealized case of a simple monopole magnetic field from the solar surface. In terms of the surface angular velocity $\Omega$ and wind mass loss rate $\dot{M}$, Weber \& Davis concluded that the total angular momentum loss rate scales as:

$$
\dot{J}=\frac{2}{3} \dot{M} \Omega R_{A}^{2},
$$

with $R_{A}$ the Alfvén radius,defined by where the radial components of the field and flow have equal energy density. However, WD did not provide a prescription for computing such an Alfvén radius even for the idealized monopole field geometry.

For any radius $r$, the energy density ratio between radial field and flow is given by

$$
\eta(r) \equiv \frac{B_{r}^{2} / 8 \pi}{\rho v_{r}^{2} / 2} .
$$


The Alfvén radius is then defined implicitly by $\eta\left(R_{A}\right) \equiv 1$. We can derive approximate explicit expressions in terms of fixed values for the equatorial field strength $B_{e q}$ at the surface radius $R_{*}$, and for the wind mass loss rate $\dot{M}$ and terminal flow speed $v_{\infty}$. Specifically, following ud-Doula \& Owocki (paper 1, 2002) and ud-Doula et al. (paper 2, 2008a), if we define here a wind magnetic confinement parameter,

$$
\eta_{*} \equiv \frac{B_{e q}^{2} R_{*}^{2}}{\dot{M} v_{\infty}}
$$

then we can write the energy density ratio in the form

$$
\eta(r)=\eta_{*}\left[\frac{r}{R_{*}}\right]^{2-2 q} \frac{v_{\infty}}{v_{r}(r)}
$$

where $q$ is the power-law exponent for radial decline of the assumed magnetic field and terminal speed $v_{\infty}$. It turns out that in the strong magnetic confinement limit $\eta_{*} \gg 1$, for a monopole field $R_{A} \sim \sqrt{\eta_{*}}$ whereas for a dipole field the scaling is significantly weaker $R_{A} \sim \eta_{*}{ }^{1 / 4}$.

\section{Key Results}

In our recent paper 3 (ud-Doula, Owocki \& Townsend (2008b)), which this poster summarizes, we examine the wind magnetic spin-down of massive stars with a rotationaligned dipole field based on previous magnetohydrodynamic (MHD) simulation parameter study presented in paper 2. Despite key differences, we find that the total angular momentum loss from these massive stars follow the general WD scaling 1.1. However, because for dipole fields the Alfvén radius has a stronger field scaling than for the idealized monopole case, the net stellar spin-down time is also significantly longer. Our numerical simulations show that this spin-down time can be expressed as:

$$
\frac{\tau_{\text {spin }}}{\tau_{\text {mass }}} \approx \frac{\frac{3}{2} k}{\left[0.29+\left(\eta_{*}+0.25\right)^{1 / 4}\right]^{2}} .
$$

where $\tau_{\text {mass }}$ is the characteristic mass loss rate $\left(=M_{*} / \dot{M}\right), k$ is the moment of inertia constant with a typical value $k \approx 0.1$. This leads to typical spin-down time of $\sim 1 \mathrm{Myr}$ for several know massive stars. Details of our full calculations can be found in ud-Doula, Owocki \& Townsend (2008b).

\section{Acknowledgements}

This work was supported by NASA Grants Chandra/TM7-8002X and NNX08AY04G.

\section{References}

ud-Doula A. \& Owocki S. P., 2002, ApJ 576, 413

ud-Doula A., Owocki S. P., \& Townsend R. H. D., 2008, MNRAS 385, 97

ud-Doula A., Owocki S. P., \& Townsend R. H. D., 2008, MNRAS, in press

Weber E. J. \& Davis L. J., 1967, ApJ 148, 217 